\title{
CRESCIMENTO MICELIAL E PARASITISMO DE Paecilomyces lilacinus SOBRE OVOS DE Meloidogyne paranaensis EM DIFERENTES TEMPERATURAS "IN VITRO"
}

\author{
"In vitro" mycelial growth and parasitism of Paecilomyces lilacinus on Meloidogyne paranaensis \\ eggs at different temperatures
}

\author{
Marina Capparelli Cadioli'1, Débora Cristina Santiago², Adriano Thibes Hoshino ${ }^{3}$, Martin Homechin ${ }^{4}$
}

\begin{abstract}
RESUMO
Paecilomyces lilacinus é um fungo de solo, parasita facultativo de ovos de nematóides, que pode crescer rapidamente "in vitro". Este trabalho teve como objetivo avaliar o crescimento micelial de $P$. lilacinus em diferentes temperaturas e selecionar os melhores isolados quanto à capacidade de parasitar ovos de Meloidogyne paranaensis. Foram avaliados isolados de $P$. lilacinus, obtidos de solos coletados na região de Londrina, PR. Para o isolamento empregou-se a técnica de diluição seriada dos solos e plaqueamento em meio de cultura semi-seletivo. A determinação do crescimento micelial e do parasitismo "in vitro" dos isolados sobre M. paranaensis foi realizada em placas de Petri contendo meio BDA. Os isolados foram incubados em B.O.D. a temperaturas de $20^{\circ} \mathrm{C}, 22,5^{\circ} \mathrm{C}, 25^{\circ} \mathrm{C}, 27,5^{\circ} \mathrm{C}$ e $30^{\circ} \mathrm{C}$. A avaliação do crescimento foi interrompida quando em um dos tratamentos a colônia do fungo atingiu a borda da placa de Petri e a determinação do parasitismo foi realizada depois de oito dias de incubação, calculando-se a porcentagem de ovos parasitados. O crescimento micelial dos isolados de $P$. lilacinus teve grande dependência da temperatura de incubação a que foram submetidos, sendo mais rápido à temperatura de $22,5^{\circ} \mathrm{C}$. Os isolados de $P$. lilacinus revelaram habilidade para infectar os ovos de $M$. paranaensis em meio BDA, principalmente na temperatura de $25^{\circ} \mathrm{C}$.
\end{abstract}

Termos para indexação: Controle Biológico, nematóide de galhas, parasita de ovos, desenvolvimento fúngico.

\begin{abstract}
Paecilomyces lilacinus is a soil fungus, facultative parasite of nematode eggs, which develops quickly "in vitro". The mycelial growth of $P$. lilacinus isolates was evaluated at different temperatures and the best isolates, regarding the capacity to parasite Meloidogyne paranaensis eggs, were chosen. P. lilacinus soil isolates from Londrina, Parana state, were evaluated. Isolation was done using serial dilution of the soils and plating it in semi-selective agar medium. The determination of mycelial growth and "in vitro" parasitism of these isolates was done using Petri plates containing potato-dextrose-agar (PDA), placed in chamber at $20^{\circ} \mathrm{C}, 22.5^{\circ} \mathrm{C}$, $25^{\circ} \mathrm{C}, 27.5^{\circ} \mathrm{C}$ or $30^{\circ} \mathrm{C}$. The evaluation started when in one of the treatments of the fungus colonies reached the edge of the Petri plate. Parasitism was determined after 8 days of incubation, calculating the percentage of parasited eggs. The mycelial growth of $P$. lilacinus isolates was greatly dependent on the temperature. The fastest growth occurred at $22.5^{\circ} \mathrm{C}$. The isolates of $P$. lilacinus were able to infect M. paranaensis eggs in PDA medium, mostly at $25^{\circ} \mathrm{C}$.
\end{abstract}

Index terms: Biological Control, root-knot nematode, egg parasite, fungal development.

\section{(Recebido em 9 de março de 2006 e aprovado em 17 de outubro de 2006)}

\section{INTRODUÇÃO}

Nematóides formadores de galhas (Meloidogyne GÖELDI) estão amplamente disseminados no Brasil e estão entre os principais problemas fitossanitários em culturas de importância econômica (CARNEIRO et al., 1996; MOURA, 1996). Dentre esses, é de grande importância a espécie M. paranaensis (CARNEIRO et al., 1996), tanto pela ampla distribuição geográfica como pela severidade dos danos causados nas diferentes culturas (CARNEIRO et al., 1996). Levantamentos realizados no Paraná e em São Paulo têm mostrado um aumento na distribuição dessa espécie em áreas cafeeiras (KRZYZANOWSKI et al., 2001; LORDELLO et al., 2001).

Os métodos mais usados para controlar fitonematóides têm sido o uso de nematicidas, variedades resistentes e rotação de culturas. No entanto, existe uma pressão da sociedade no sentido da substituição dos atuais nematicidas por princípios ativos ou por táticas de controle

\footnotetext{
'Engenheira Agrônoma, Pós-Graduanda, Mestrado em Agronomia - Universidade Estadual de Londrina/UEL - Centro de Ciências Agrárias Departamento de Agronomia - Rodovia Celso Garcia Cid, PR 445 Km 380 - Campus Universitário - Cx. P. 6001 - $86051-990$ - Londrina, PR marinacadioli@hotmail.com

${ }^{2}$ Engenheira Agrônoma, Professora Doutora - Universidade Estadual de Londrina/UEL - Centro de Ciências Agrárias - Departamento de Agronomia Rodovia Celso Garcia Cid, PR 445 Km 380 - Campus Universitário - Cx. Postal 6001 - 86051-990 - Londrina, PR - santiago@uel.br

${ }^{3}$ Graduando do Curso de Agronomia - Universidade Estadual de Londrina/UEL - Centro de Ciências Agrárias - Departamento de Agronomia Rodovia Celso Garcia Cid, PR 445 Km 380 - Campus Universitário - Cx. P. 6001 - 86051-990 - Londrina, PR - hoshinoagro@gmail.com ${ }^{4}$ Engenheiro Agrônomo, Professor Doutor - Universidade Estadual de Londrina/UEL - Centro de Ciências Agrárias - Departamento de Agronomia Rodovia Celso Garcia Cid, PR 445 Km 380 - Campus Universitário - Cx. P. 6001 - 86051-990 - Londrina, PR - homechin@uel.br
} 
ecologicamente mais recomendáveis. Esse fato tem levado à busca de métodos alternativos para o controle de fitonematóides, principalmente para os gêneros de maior importância, a exemplo de Meloidogyne (CARNEIRO, 1992).

A supressão dos nematóides devido à atuação de antagonistas, observada em alguns solos, e a não recomendação de alguns nematicidas aumentaram o interesse pelo controle biológico com o emprego de fungos nematófagos (FERRAZ \& SANTOS, 1995). Muitos fungos têm demonstrado capacidade de parasitar fitonematóides (CARNEIRO, 1992; MANKAU, 1980).

Paecilomyces lilacinus (THOM) SAMSON é um fungo de solo que tem se mostrado efetivo no controle de espécies de Meloidogyne (CAMPOS \& CAMPOS, 1996, 1997; KERRY, 1990). Foi reportado pela primeira vez como parasita em 1976 por Jatala, quando observou a presença deste infectando ovos de M. incognita (Kofoid \& White, 1919) Chitwood (1949) e Globodera pallida (Stone, 1973) Behrens (1975) sobre raízes de batatas (Solanum tuberosum L.) no Peru (JATALA, 1986). No Brasil, o primeiro relato de P. lilacinus como parasita de ovos de Meloidogyne sp. foi feito por Freire \& Bridge (1985), em raízes de pimenta-doreino (Piper nigrum L.) cv. Singapura.

$P$. lilacinus é um anamorfo de ascomiceto da ordem Eurotiales, encontrado em diferentes regiões do mundo e tem sido observado com maior freqüência em regiões quentes. Sua presença tem sido detectada em diferentes tipos de hospedeiros e solos, cultivados ou não (FARIA \& TIGANO, 1996; SOSA-GOMEZ, 2002), sendo mais comuns em profundidades variando de 0-40 cm (CARNEIRO, 1986). É um parasita facultativo de ovos de nematóides que pode crescer rapidamente "in vitro" e a sua sobrevivência no solo não depende da presença dos nematóides (CARNEIRO, 1992).

A seleção de isolados, quanto ao parasistismo, é de extrema importância na busca de microrganismos eficientes como agentes de controle biológico e adaptados a diferentes regiões. Novaretti et al. (1986), na cultura de cana-de-açúcar e Hewlett et al. (1988) em fumo, contestaram a eficiência desse fungo em condições de campo, provavelmente devido à inadequação dos métodos de aplicação, de produção de conídios do fungo e de avaliação dos ensaios (KERRY, 1990) e não adaptação do isolado a diferentes condições e tipos de solo (CARNEIRO, 1992).

Dentro de uma espécie fúngica existem variações quanto à capacidade de colonizar os ovos de nematóides. Rodríguez-Kábana et al. (1984) e Stirling \& West (1991) observaram que isolados de Verticillium chlamydosporium GODDARD e de $P$. lilacinus apresentavam variabilidade quanto ao grau de parasitismo, sendo esta mais acentuada para P. lilacinus.

Para que se possa saber se os isolados de $P$. lilacinus podem, ou não, ser utilizados no controle de nematóides, é necessário definir, primeiro, quais as melhores condições de multiplicação desse fungo, principalmente no que se refere à temperatura, uma vez que esse microrganismo ficará exposto à variação térmica que ocorre no solo durante o ano (FIORETTO \& VILLACORTA, 1981). Embora resultados encorajadores sejam observados em condições brasileiras (COSTA \& CAMPOS, 1997; FREITAS et al., 1999; MIZOBUTSI et al., 2000), informações básicas sobre o comportamento de $P$. lilacinus como parasita de nematóides de galhas, em diferentes condições climáticas e ambientais do solo, são necessárias para que seu emprego na agricultura seja recomendado, especialmente no controle de $M$. paranaensis, nematóide carente de informações com relação ao seu manejo.

Portanto, realizou-se este trabalho com o objetivo de avaliar o crescimento micelial de $P$. lilacinus em diferentes temperaturas $\left(20^{\circ} \mathrm{C}, 22,5^{\circ} \mathrm{C}, 25^{\circ} \mathrm{C}, 27,5^{\circ} \mathrm{C}\right.$ e $\left.30^{\circ} \mathrm{C}\right)$ e selecionar os melhores isolados quanto a capacidade de parasitar ovos de M. paranaensis "in vitro".

\section{MATERIAL E MÉTODOS}

Foram avaliados 31 isolados de $P$. lilacinus, obtidos de amostras de solos coletadas em áreas cultivadas com café, pastagem, eucalipto e milho, na região de Londrina PR, no período de março a maio de 2005.

Para o isolamento de $P$. lilacinus foi empregada a técnica de diluição seriada dos solos e plaqueamento em meio de cultura semi-seletivo de Alves et al. (1998), com pequenas modificações, composto por $20 \mathrm{~g}$ de farinha de aveia, $20 \mathrm{~g}$ de ágar, $300 \mathrm{mg}$ de Venturol® (dodine $650 \mathrm{~g}^{\mathrm{kg}} \mathrm{kg}^{-1}$ ), $50 \mathrm{mg}$ de solução de violeta genciana, $5 \mathrm{mg}$ de Tetraciclina (cloridrato de tetraciclina $300 \mathrm{mg}$ ) em $1000 \mathrm{~mL}$ de água destilada. Após a obtenção e identificação dos isolados de $P$. lilacinus, estes foram repicados para meio de batatadextrose-ágar (BDA) em tubos de ensaio, catalogados e armazenados em geladeira.

A determinação do crescimento micelial e do parasitismo "in vitro" dos isolados sobre $M$. paranaensis foi realizada em placas de Petri contendo $15 \mathrm{~mL}$ de meio ágar-água (1,5\%). Discos de $5 \mathrm{~mm}$ de diâmetro dos isolados fúngicos, obtidos de cultivo puro em BDA, foram transferidos para o centro de cada placa. Na testemunha foi transferido apenas um disco de $5 \mathrm{~mm}$ de meio de BDA sem o fungo. Ao redor dos discos foram colocadas três 
massas de ovos de M. paranaensis, obtidas a partir de uma população pura multiplicada em plantas de tomateiro (Lycopersicon esculentum L.) cv. Santa Cruz durante 45 dias sob condições de casa-de-vegetação.

As placas foram incubadas em câmaras do tipo B.O.D. às temperaturas de $20^{\circ} \mathrm{C}, 22,5^{\circ} \mathrm{C}, 25^{\circ} \mathrm{C}, 27,5^{\circ} \mathrm{C}$ e $30^{\circ} \mathrm{C}$, com 12 horas de luz e 12 horas de escuro. $\mathrm{O}$ crescimento micelial foi acompanhado diariamente, medindo-se o diâmetro da colônia do fungo em dois sentidos perpendiculares entre si. A avaliação foi interrompida quando em um dos tratamentos a colônia do fungo atingiu a borda da placa de Petri.

A determinação do parasitismo foi realizada depois de oito dias de incubação. Para tanto, discos eqüidistantes de $9 \mathrm{~mm}$ de diâmetro foram retirados ao redor das massas de ovos de cada placa e foram colocados em seqüência sobre uma lâmina para microscopia. Sobre os discos foi acrescentada uma gota de lactofenol, contendo azul de algodão, e em microscópio ótico, sob aumento de 100 vezes, foi avaliada a porcentagem de ovos parasitados em relação ao número total.

Para análise estatística foi utilizado o modelo fatorial 31 x 5 (isolados x temperaturas) com 10 repetições, cinco para a obtenção do crescimento micelial e cinco para o teste de parasitismo, e as médias dos tratamentos foram comparadas por meio dos testes de Scott-knot (para isolado) e de Tukey (para temperatura) ao nível de 5\% de probabilidade.

\section{RESULTADOS E DISCUSSÃO}

A variância para as temperaturas testadas mostrou significância, o que indica que o crescimento micelial dos isolados de $P$. lilacinus teve grande dependência da temperatura de incubação a que foram submetidos (Tabela 1). $\mathrm{Na}$ temperatura de $20^{\circ} \mathrm{C}$ foi observado que os isolados Pae 03, 05, 10, 12 e 20 apresentaram desenvolvimento micelial superior aos demais no período considerado. $\mathrm{Na}$ temperatura de $22,5^{\circ} \mathrm{C}$, os melhores crescimentos foram observados para os isolados Pae 03, 06, 07, 09, 10, 11 e 13. $\mathrm{Na}$ temperatura de $25^{\circ} \mathrm{C}$, os isolados Pae 08 e 22 se destacaram com crescimento superior aos demais. Já para a temperatura de $27,5^{\circ} \mathrm{C}$, o isolado Pae 21 teve melhor crescimento micelial e os isolados que se destacaram na temperatura de $30^{\circ} \mathrm{C}$ foram Pae 22,24 e 30 .

Com relação ao desenvolvimento micelial observouse um aspecto interessante na temperatura de $22,5^{\circ} \mathrm{C}$, em que os isolados Pae 03, 06, 07, 09, 10, 11 e 13 apresentaram um crescimento muito mais rápido em comparação às outras temperaturas avaliadas, chegando a atingirem a borda das placas ao quinto dia de incubação. Estes resultados diferem dos obtidos por Fioretto \& Villacorta (1981), os quais observaram maior crescimento de $P$. lilacinus nas temperaturas de 24 e $25^{\circ} \mathrm{C}$. No entanto, estes autores estudaram o comportamento apenas de um isolado proveniente do Peru.

Para os isolados Pae 02, 23 e 31 a temperatura não influenciou de maneira significativa o crescimento micelial. As temperaturas de 20 e $22,5^{\circ} \mathrm{C}$ foram as melhores para o desenvolvimento do isolado Pae 05. Os isolados Pae 04, 09 e 16 tiveram crescimento semelhante tanto a 22,5 quanto a $25^{\circ} \mathrm{C}$. Já os isolados Pae 18, 27 e 29 apresentaram crescimento semelhante nas temperaturas de $22,5^{\circ} \mathrm{C}$ e $27,5^{\circ} \mathrm{C}$. As temperaturas de 25 e $27,5^{\circ} \mathrm{C}$ foram as melhores para o isolado Pae 25 e as temperaturas de 25 e $30^{\circ} \mathrm{C}$ para os isolados Pae 22 e 24. Os isolados Pae 01, 15, 19, 20 e 30 apresentaram resultados variáveis em função das temperaturas. Já o isolado Pae 21 teve maior crescimento na temperatura de $27,5^{\circ} \mathrm{C}$ e os isolados Pae 12 e 26 apresentaram maior desenvolvimento micelial nas temperaturas de $25^{\circ} \mathrm{C}$ e $27,5^{\circ} \mathrm{C}$, respectivamente. E os demais apresentaram melhor crescimento micelial na temperatura de $22,5^{\circ} \mathrm{C}$. Estes resultados corroboram com o que foi citado por Felli et al. (1985) e Jatala (1986). E, por isso, a eficiência e a adaptabilidade de $P$. lilacinus no controle de nematóides em diferentes condições climáticas e ambientais do solo, ainda necessita ser melhor explorada. Portanto, o presente estudo pode ser importante na recomendação dos isolados em função das condições climáticas de cada região.

Quanto à capacidade de parasitismo, os isolados de $P$. lilacinus revelaram habilidade para infectar os ovos de $M$. paranaensis em meio BDA (Tabela 2). Na temperatura de $20^{\circ} \mathrm{C}$, os isolados mais agressivos aos ovos foram Pae 03, $05,09,10,12,13,17,20,26,28,29$ e 30, com percentuais de parasitismo que variaram de 55,98 a 95,23\%. Na temperatura de $22,5^{\circ} \mathrm{C}$ os isolados que apresentaram maior porcentagem de parasitismo foram Pae 03, 04, 05, 06, 07, 10, 12, 13, 15, 18, $20,21,22,23,24,25,26,27,28,30$ e 31 , com percentuais variando de 69,98 a $98,66 \%$. Na temperatura de $25^{\circ} \mathrm{C}$, os isolados Pae 13, 17, 22 e 26 diferiram estatisticamente dos demais, apresentando os menores percentuais de ovos parasitados. Estes percentuais variaram entre 80,39 e 87,15\%, no entanto foram bem superiores àqueles menores observados nas temperaturas de 20 e $22,5^{\circ} \mathrm{C}$. Os isolados mais agressivos na temperatura de $27,5^{\circ} \mathrm{C}$ foram Pae 05,09 , $11,14,20,21,22$ e 26 com percentuais variando de 81,00 a $95,86 \%$. Na temperatura de $30^{\circ} \mathrm{C}$, com exceção dos isolados Pae 04, 09, 13, 14, 17 e 29, todos os demais apresentaram percentuais de parasitismo superiores a 79,20\%. 
TABELA 1 -Crescimento micelial de isolados de Paecilomyces lilacinus "in vitro" em diferentes temperaturas de incubação.

\begin{tabular}{|c|c|c|c|c|c|}
\hline \multirow{2}{*}{ Isolado } & \multicolumn{5}{|c|}{ Temperatura } \\
\hline & $20^{\circ} \mathrm{C}$ & $22,5^{\circ} \mathrm{C}$ & $25^{\circ} \mathrm{C}$ & $27,5^{\circ} \mathrm{C}$ & $30^{\circ} \mathrm{C}$ \\
\hline Pae01 & $3,03 \mathrm{C}^{1} \mathrm{ab}^{2}$ & $2,33 \mathrm{D} \mathrm{b}$ & $1,53 \mathrm{E} \mathrm{b}$ & $2,88 \mathrm{D} a b$ & $4,28 \mathrm{D}$ a \\
\hline $\mathrm{Pae} 02$ & $2,00 \mathrm{D} \mathrm{a}$ & $3,25 \mathrm{D}$ a & $1,94 \mathrm{E} \mathrm{a}$ & $3,40 \mathrm{C} \mathrm{a}$ & $3,00 \mathrm{E}$ a \\
\hline Pae03 & $4,95 \mathrm{~A} \mathrm{~b}$ & $8,25 \mathrm{~A} \mathrm{a}$ & $3,20 \mathrm{D} \mathrm{c}$ & $3,05 \mathrm{C} \mathrm{c}$ & $5,10 \mathrm{C} \mathrm{b}$ \\
\hline $\mathrm{Pae} 04$ & $3,83 \mathrm{~B} \mathrm{bc}$ & $5,45 \mathrm{~B} \mathrm{ab}$ & $5,95 \mathrm{~B}$ a & $2,75 \mathrm{D} \mathrm{cd}$ & $1,70 \mathrm{~F} \mathrm{~d}$ \\
\hline Pae05 & $4,80 \mathrm{~A} \mathrm{a}$ & $6,20 \mathrm{~B} \mathrm{a}$ & $2,84 \mathrm{D} \mathrm{b}$ & $1,66 \mathrm{D}$ bc & $1,13 \mathrm{~F} \mathrm{c}$ \\
\hline Pae06 & $4,18 \mathrm{~B} \mathrm{~b}$ & $7,40 \mathrm{~A} \mathrm{a}$ & $3,52 \mathrm{D} \mathrm{b}$ & $3,05 \mathrm{C} \mathrm{bc}$ & $1,56 \mathrm{~F} \mathrm{c}$ \\
\hline Pae07 & $3,90 \mathrm{~B} \mathrm{~b}$ & $7,75 \mathrm{~A} \mathrm{a}$ & $4,49 \mathrm{C} \mathrm{b}$ & $2,20 \mathrm{D} \mathrm{c}$ & $1,80 \mathrm{~F} \mathrm{c}$ \\
\hline $\mathrm{Pae} 08$ & $1,84 \mathrm{D} \mathrm{c}$ & $6,25 \mathrm{~B}$ a & $2,81 \mathrm{D}$ bc & $3,30 \mathrm{C} \mathrm{bc}$ & $3,74 \mathrm{D} \mathrm{b}$ \\
\hline Pae09 & $4,46 \mathrm{~B} \mathrm{~b}$ & $8,35 \mathrm{~A} \mathrm{a}$ & $7,23 \mathrm{~A} \mathrm{a}$ & $3,20 \mathrm{C} \mathrm{b}$ & $1,06 \mathrm{~F} \mathrm{c}$ \\
\hline Pae10 & $5,00 \mathrm{~A} \mathrm{~b}$ & $7,80 \mathrm{~A} \mathrm{a}$ & $3,28 \mathrm{D} \mathrm{c}$ & $2,40 \mathrm{D} \mathrm{c}$ & $5,22 \mathrm{Cb}$ \\
\hline Pae11 & $3,50 \mathrm{Cb}$ & $7,10 \mathrm{~A} \mathrm{a}$ & $2,67 \mathrm{D} \mathrm{b}$ & $2,26 \mathrm{D} \mathrm{b}$ & $3,24 \mathrm{E} \mathrm{b}$ \\
\hline Pae12 & $5,57 \mathrm{~A} \mathrm{a}$ & $5,10 \mathrm{C} \mathrm{a}$ & $5,57 \mathrm{~B}$ a & $1,90 \mathrm{D} \mathrm{b}$ & $1,81 \mathrm{~F} \mathrm{~b}$ \\
\hline Pae13 & $4,00 \mathrm{~B} \mathrm{~b}$ & $8,15 \mathrm{~A} \mathrm{a}$ & $2,45 \mathrm{E} \mathrm{bc}$ & $2,40 \mathrm{D} b c$ & $1,79 \mathrm{~F} \mathrm{c}$ \\
\hline Pae14 & $3,61 \mathrm{C} \mathrm{b}$ & $6,75 \mathrm{~B}$ a & $4,28 \mathrm{C} \mathrm{b}$ & $4,12 \mathrm{C} \mathrm{b}$ & $1,83 \mathrm{~F} \mathrm{c}$ \\
\hline Pae15 & $3,57 \mathrm{Cb}$ & $6,65 \mathrm{~B} \mathrm{a}$ & $5,27 \mathrm{~B} \mathrm{~b}$ & $2,80 \mathrm{D} \mathrm{b}$ & $5,70 \mathrm{~B}$ a \\
\hline Pae16 & $3,30 \mathrm{C} \mathrm{bc}$ & $4,80 \mathrm{C} \mathrm{ab}$ & $5,89 \mathrm{~B}$ a & $2,10 \mathrm{D} \mathrm{c}$ & $1,75 \mathrm{~F} \mathrm{c}$ \\
\hline Pae17 & $3,64 \mathrm{C} \mathrm{b}$ & $6,30 \mathrm{~B} \mathrm{a}$ & $3,62 \mathrm{D} b \mathrm{c}$ & $1,90 \mathrm{D} \mathrm{d}$ & $1,95 \mathrm{~F} \mathrm{~cd}$ \\
\hline Pae18 & $4,08 \mathrm{~B} \mathrm{~b}$ & $6,70 \mathrm{~B}$ a & $2,71 \mathrm{D} b c$ & $6,05 \mathrm{~B}$ a & $2,33 \mathrm{E} \mathrm{c}$ \\
\hline Pae19 & $4,66 \mathrm{~B} \mathrm{a}$ & $2,46 \mathrm{D} \mathrm{b}$ & $5,97 \mathrm{~B}$ a & $2,55 \mathrm{D} \mathrm{b}$ & $5,85 \mathrm{~B}$ a \\
\hline Pae20 & $6,00 \mathrm{~A} \mathrm{a}$ & $6,05 \mathrm{~B}$ a & $3,69 \mathrm{D} \mathrm{b}$ & $5,50 \mathrm{~B}$ a & $5,55 \mathrm{~B}$ a \\
\hline Pae21 & $3,85 \mathrm{~B} \mathrm{~b}$ & $5,40 \mathrm{~B} \mathrm{~b}$ & $4,86 \mathrm{C} \mathrm{b}$ & $7,35 \mathrm{~A}$ a & $4,63 \mathrm{C} \mathrm{b}$ \\
\hline Pae22 & $4,55 \mathrm{~B} \mathrm{c}$ & $4,90 \mathrm{C} \mathrm{c}$ & $7,65 \mathrm{~A}$ a & $5,65 \mathrm{~B} b \mathrm{c}$ & $6,68 \mathrm{~A} \mathrm{ab}$ \\
\hline Pae23 & $4,55 \mathrm{~B} \mathrm{a}$ & $5,20 \mathrm{C} \mathrm{a}$ & $4,35 \mathrm{C} \mathrm{a}$ & $5,70 \mathrm{~B}$ a & $4,70 \mathrm{C} \mathrm{a}$ \\
\hline Pae24 & $4,32 \mathrm{~B} \mathrm{bc}$ & $4,85 \mathrm{C} \mathrm{bc}$ & $5,82 \mathrm{~B} \mathrm{ab}$ & $3,80 \mathrm{C} \mathrm{c}$ & $7,45 \mathrm{~A}$ a \\
\hline Pae25 & $2,10 \mathrm{D} \mathrm{b}$ & $1,02 \mathrm{D} \mathrm{b}$ & $5,46 \mathrm{~B}$ a & $5,00 \mathrm{~B}$ a & $2,60 \mathrm{E} \mathrm{b}$ \\
\hline Pae26 & $3,15 \mathrm{C} \mathrm{ab}$ & $4,15 \mathrm{C} \mathrm{a}$ & $3,15 \mathrm{D} a b$ & $3,80 \mathrm{C} \mathrm{a}$ & $2,03 \mathrm{~F} \mathrm{~b}$ \\
\hline Pae27 & $3,32 \mathrm{C} \mathrm{bc}$ & $4,65 \mathrm{C} \mathrm{ab}$ & $2,82 \mathrm{D} \mathrm{c}$ & $5,55 \mathrm{~B}$ a & $2,23 \mathrm{E} \mathrm{c}$ \\
\hline Pae28 & $4,00 \mathrm{~B} \mathrm{~b}$ & $5,85 \mathrm{~B} \mathrm{a}$ & $2,91 \mathrm{D} b \mathrm{cc}$ & $4,05 \mathrm{C} \mathrm{b}$ & $2,02 \mathrm{~F} \mathrm{c}$ \\
\hline Pae29 & $2,10 \mathrm{D} a b c$ & $3,30 \mathrm{D} \mathrm{ab}$ & $1,72 \mathrm{E} \mathrm{bc}$ & $3,60 \mathrm{C} \mathrm{a}$ & $1,54 \mathrm{~F} \mathrm{c}$ \\
\hline Pae30 & $3,25 \mathrm{C} \mathrm{b}$ & $6,70 \mathrm{~B}$ a & $3,31 \mathrm{D} \mathrm{b}$ & $4,80 \mathrm{~B} \mathrm{~b}$ & $6,59 \mathrm{~A} a$ \\
\hline Pae31 & $2,80 \mathrm{C} \mathrm{a}$ & $2,60 \mathrm{D}$ a & $2,90 \mathrm{D}$ a & $2,60 \mathrm{D} \mathrm{a}$ & $4,26 \mathrm{D}$ a \\
\hline $\mathrm{CV}(\%)$ & 18,6 & 21,06 & 14,45 & 40,47 & 19,15 \\
\hline
\end{tabular}

${ }^{1}$ Médias seguidas pelas mesmas letras maiúsculas nas colunas, não diferem entre si, pelo teste de Scott-knot ao nível de $5 \%$ de probabilidade. ${ }^{2}$ Médias seguidas pelas mesmas letras minúsculas nas linhas, não diferem entre si, pelo teste de Tukey ao nível de 5\% de probabilidade.

Em se considerando o parasitismo dos isolados, nas temperaturas de 25 e $30^{\circ} \mathrm{C}$, foram encontrados os maiores percentuais de parasitismo dos ovos, a exceção dos isolados Pae 04, 09, 13, 14, 17, 29 e 30, os quais na temperatura de $30^{\circ} \mathrm{C}$ apresentaram percentuais semelhantes aos observados em $20^{\circ} \mathrm{C}$. Para os isolados Pae 07, 18 e 28 em $25^{\circ} \mathrm{C}$, e Pae 24 na temperatura de $30^{\circ} \mathrm{C}, 100 \%$ dos ovos encontravam-se parasitados.

$\mathrm{Na}$ temperatura de $22,5^{\circ} \mathrm{C}$ houve grande variação na porcentagem de parasitismo, apesar do rápido crescimento micelial observado nesta temperatura, sendo similar ao resultado obtido por Freitas et al. (1995), comparando 19 isolados de $P$. lilacinus de diferentes procedências quanto ao parasitismo de ovos de $M$. javanica, os quais obtiveram $100 \%$ de parasitismo pelos isolados procedentes da Itália e do Peru e, $70 \%$ pelo isolado originário da França. Já para os isolados brasileiros houve variação de 2 a $69 \%$ de ovos parasitados. Da mesma forma, Santos et al. (1992) observou resultado diferenciado no parasitismo de $M$. incognita por diferentes isolados de $P$. lilacinus. Esta variabilidade pode ser devida à adaptação seletiva aos vários fatores edáficos em sua origem geográfica, tal como tipo de solo ou temperatura ambiente. 
TABELA 2 - Parasitismo de ovos de Meloidogyne paranaensis "in vitro" por isolados de Paecilomyces lilacinus em diferentes temperaturas de incubação.

\begin{tabular}{|c|c|c|c|c|c|}
\hline \multirow{2}{*}{ Isolado } & \multicolumn{5}{|c|}{ Temperatura } \\
\hline & $20^{\circ} \mathrm{C}$ & $22,5^{\circ} \mathrm{C}$ & $25^{\circ} \mathrm{C}$ & $27,5^{\circ} \mathrm{C}$ & $30^{\circ} \mathrm{C}$ \\
\hline Pae01 & $47,82 \mathrm{~B} \mathrm{c}^{2}$ & $62,42 \mathrm{~B} \mathrm{bc}$ & $98,90 \mathrm{~A} \mathrm{a}$ & $37,00 \mathrm{C} \mathrm{c}$ & $89,45 \mathrm{~A}$ ab \\
\hline $\mathrm{Pae} 02$ & $21,88 \mathrm{~B} \mathrm{bc}$ & $42,00 \mathrm{C} \mathrm{b}$ & $92,28 \mathrm{~A} \mathrm{a}$ & $8,33 \mathrm{D} \mathrm{c}$ & $82,56 \mathrm{~A} \mathrm{a}$ \\
\hline $\mathrm{Pae} 03$ & $64,07 \mathrm{~A} \mathrm{~b}$ & $73,63 \mathrm{~A} \mathrm{ab}$ & $96,18 \mathrm{~A}$ a & $15,33 \mathrm{D} \mathrm{c}$ & $95,35 \mathrm{~A} \mathrm{a}$ \\
\hline $\mathrm{Pae} 04$ & $32,14 \mathrm{~B} \mathrm{c}$ & $69,98 \mathrm{~A} \mathrm{~b}$ & $98,89 \mathrm{~A} \mathrm{a}$ & $45,32 \mathrm{C} \mathrm{bc}$ & $51,94 \mathrm{C} \mathrm{bc}$ \\
\hline $\mathrm{Pae} 05$ & $62,57 \mathrm{~A} \mathrm{~b}$ & $93,55 \mathrm{~A} \mathrm{a}$ & $93,62 \mathrm{~A} \mathrm{a}$ & $92,00 \mathrm{~A} \mathrm{a}$ & $85,49 \mathrm{~A}$ ab \\
\hline Pae06 & $34,50 \mathrm{~B} \mathrm{~b}$ & $98,00 \mathrm{~A} \mathrm{a}$ & $97,72 \mathrm{~A} \mathrm{a}$ & $36,66 \mathrm{Cb}$ & $88,29 \mathrm{~A} \mathrm{a}$ \\
\hline $\mathrm{Pae} 07$ & $44,51 \mathrm{~B} \mathrm{~b}$ & $97,06 \mathrm{~A} \mathrm{a}$ & $100,00 \mathrm{~A} \mathrm{a}$ & $73,10 \mathrm{~B} \mathrm{a}$ & $92,26 \mathrm{~A} \mathrm{a}$ \\
\hline Pae08 & $23,21 \mathrm{~B} \mathrm{~b}$ & $33,28 \mathrm{C} \mathrm{b}$ & $95,62 \mathrm{~A} \mathrm{a}$ & $11,66 \mathrm{D} \mathrm{b}$ & $89,13 \mathrm{~A}$ a \\
\hline Pae09 & $79,40 \mathrm{~A} \mathrm{ab}$ & $40,66 \mathrm{C} \mathrm{c}$ & $99,50 \mathrm{~A} \mathrm{a}$ & $90,99 \mathrm{~A} \mathrm{a}$ & $59,98 \mathrm{~B} \mathrm{bc}$ \\
\hline Pae10 & $77,36 \mathrm{~A} \mathrm{ab}$ & $96,10 \mathrm{~A}$ a & $98,83 \mathrm{~A}$ a & $60,38 \mathrm{~B} \mathrm{~b}$ & $95,63 \mathrm{~A}$ a \\
\hline Pae11 & $33,79 \mathrm{~B} \mathrm{c}$ & $65,33 \mathrm{~B} \mathrm{~b}$ & $97,73 \mathrm{~A} \mathrm{a}$ & $88,33 \mathrm{~A}$ ab & $87,40 \mathrm{~A}$ ab \\
\hline Pae12 & $94,50 \mathrm{~A} \mathrm{a}$ & $92,18 \mathrm{~A} \mathrm{a}$ & $98,95 \mathrm{~A} \mathrm{a}$ & 49,66 B b & $91,74 \mathrm{~A} a$ \\
\hline Pae13 & $70,19 \mathrm{~A} \mathrm{abc}$ & $81,66 \mathrm{~A}$ ab & $87,15 \mathrm{~B}$ a & $58,64 \mathrm{~B} \mathrm{bc}$ & $45,86 \mathrm{C} \mathrm{c}$ \\
\hline Pae14 & $47,84 \mathrm{~B} \mathrm{~b}$ & $19,99 \mathrm{C} \mathrm{c}$ & $94,58 \mathrm{~A} \mathrm{a}$ & $81,00 \mathrm{~A} \mathrm{a}$ & $47,74 \mathrm{C} \mathrm{b}$ \\
\hline Pae15 & $23,20 \mathrm{~B} \mathrm{c}$ & $86,11 \mathrm{~A} \mathrm{ab}$ & $91,82 \mathrm{~A} \mathrm{a}$ & $63,72 \mathrm{~B} \mathrm{~b}$ & $89,90 \mathrm{~A} \mathrm{ab}$ \\
\hline Pae16 & $21,88 \mathrm{~B} \mathrm{c}$ & $59,42 \mathrm{~B} \mathrm{~b}$ & $99,57 \mathrm{~A}$ a & $7,66 \mathrm{D} \mathrm{c}$ & $80,24 \mathrm{~A} a b$ \\
\hline Pae17 & $56,25 \mathrm{~A}$ ab & $53,78 \mathrm{~B} \mathrm{ab}$ & $80,39 \mathrm{~B}$ a & $41,00 \mathrm{C} \mathrm{b}$ & $70,42 \mathrm{~B} \mathrm{a}$ \\
\hline Pae18 & $37,32 \mathrm{~B} \mathrm{~b}$ & $94,78 \mathrm{~A} \mathrm{a}$ & $100,00 \mathrm{~A} \mathrm{a}$ & $50,72 \mathrm{~B} \mathrm{~b}$ & $79,78 \mathrm{~A}$ a \\
\hline Pae19 & $33,72 \mathrm{~B} \mathrm{~b}$ & $34,99 \mathrm{C} \mathrm{b}$ & $98,86 \mathrm{~A} \mathrm{~b}$ & $12,83 \mathrm{D} \mathrm{a}$ & $97,47 \mathrm{~A} \mathrm{a}$ \\
\hline Pae20 & $95,23 \mathrm{~A} \mathrm{a}$ & $86,66 \mathrm{~A}$ a & $98,42 \mathrm{~A}$ a & $95,86 \mathrm{~A}$ a & $95,66 \mathrm{~A}$ a \\
\hline Pae21 & $44,51 \mathrm{~B} \mathrm{~b}$ & $80,32 \mathrm{~A}$ a & $98,19 \mathrm{~A} \mathrm{a}$ & $88,39 \mathrm{~A} \mathrm{a}$ & $98,89 \mathrm{~A} \mathrm{a}$ \\
\hline Pae22 & $47,46 \mathrm{~B} \mathrm{~b}$ & $96,66 \mathrm{~A}$ a & $80,54 \mathrm{~B}$ a & $86,33 \mathrm{~A}$ a & $96,60 \mathrm{~A}$ a \\
\hline Pae23 & $48,58 \mathrm{~B} \mathrm{~b}$ & $95,00 \mathrm{~A}$ a & $91,74 \mathrm{~A}$ a & $5,66 \mathrm{D} \mathrm{c}$ & $95,92 \mathrm{~A} \mathrm{a}$ \\
\hline $\mathrm{Pae} 24$ & $51,06 \mathrm{~B} \mathrm{~b}$ & $95,76 \mathrm{~A} \mathrm{a}$ & $94,30 \mathrm{~A} \mathrm{a}$ & $14,66 \mathrm{D} \mathrm{c}$ & $100,00 \mathrm{~A} \mathrm{a}$ \\
\hline $\mathrm{Pae} 25$ & $20,91 \mathrm{~B} \mathrm{c}$ & $78,32 \mathrm{~A} \mathrm{ab}$ & $97,16 \mathrm{~A} \mathrm{a}$ & $56,33 \mathrm{~B} \mathrm{~b}$ & $81,72 \mathrm{~A} a b$ \\
\hline Pae26 & $55,98 \mathrm{~A} \mathrm{~b}$ & $91,66 \mathrm{~A} \mathrm{a}$ & $82,15 \mathrm{~B}$ ab & $89,06 \mathrm{~A} \mathrm{a}$ & $93,34 \mathrm{~A}$ a \\
\hline Pae27 & $34,69 \mathrm{~B} \mathrm{~b}$ & $93,06 \mathrm{~A}$ a & $99,49 \mathrm{~A} \mathrm{a}$ & $52,66 \mathrm{~B} \mathrm{~b}$ & $87,22 \mathrm{~A}$ a \\
\hline Pae28 & $56,78 \mathrm{~A} \mathrm{~b}$ & $85,00 \mathrm{~A}$ a & $100,00 \mathrm{~A} \mathrm{a}$ & $16,33 \mathrm{D} \mathrm{c}$ & $89,05 \mathrm{~A}$ a \\
\hline Pae29 & $58,59 \mathrm{~A} \mathrm{~b}$ & $65,00 \mathrm{~B} \mathrm{~b}$ & $99,03 \mathrm{~A}$ a & $54,78 \mathrm{~B} \mathrm{~b}$ & $43,68 \mathrm{C} \mathrm{b}$ \\
\hline Pae30 & $65,51 \mathrm{~A} \mathrm{~b}$ & $98,66 \mathrm{~A}$ a & $99,95 \mathrm{~A} \mathrm{a}$ & $70,32 \mathrm{~B} \mathrm{~b}$ & $79,20 \mathrm{~A} \mathrm{ab}$ \\
\hline Pae31 & $31,68 \mathrm{~B} \mathrm{~b}$ & $97,00 \mathrm{~A} \mathrm{a}$ & $99,87 \mathrm{~A} \mathrm{a}$ & $10,33 \mathrm{D} \mathrm{b}$ & $81,66 \mathrm{~A} \mathrm{a}$ \\
\hline $\mathrm{CV}(\%)$ & 46,16 & 21,15 & 5,56 & 34,44 & 15,47 \\
\hline
\end{tabular}

${ }^{1}$ Médias seguidas pelas mesmas letras maiúsculas, nas colunas, não diferem entre si, pelo teste de Scott-knot ao nível de $5 \%$ de probabilidade. ${ }^{2}$ Médias seguidas pelas mesmas letras minúsculas, nas linhas, não diferem entre si, pelo teste de Tukey ao nível de 5\% de probabilidade.

Variações quanto à capacidade de colonizar os ovos de nematóides são comumente observadas dentro de uma mesma espécie fúngica. Rodríguez--Kábana et al. (1984) e Stirling \& West (1991) observaram que isolados de $V$. chlamydosporium e de $P$. lilacinus apresentavam variabilidade na patogenicidade, sendo esta mais acentuada para $P$. lilacinus.

Mizobutsi et al. (2000) verificaram que o fungo $P$. lilacinus parasitou $77 \%$ dos ovos de $M$. javanica (Treub,
1885) Chitwood (1949), enquanto que a maioria dos isolados de outros fungos testados mostrou-se pouco eficaz no parasitismo. O fato das fêmeas adultas de Meloidogyne concentrarem seus ovos em uma matriz gelatinosa pode facilitar o desenvolvimento do $P$. lilacinus e o parasitismo dos ovos. Segundo Jatala (1986), fungos parasitas de ovos são mais eficientes na redução da população de nematóides em comparação com aqueles que atuam como endoparasitas e predadores. Também são considerados 
os agentes mais promissores para o biocontrole, uma vez que impedem ou reduzem a formação de ovos pelos nematóides (KERRY et al., 1982).

Para que se possa saber se os isolados de $P$. lilacinus podem, ou não, ser utilizados no controle de nematóides, é necessário definir, primeiro, quais as melhores condições de multiplicação desse fungo, principalmente no que se refere à temperatura, uma vez que esse microrganismo ficará exposto à variação térmica que ocorre no solo durante o ano (FIORETTO \& VILLACORTA, 1981).

\section{CONCLUSÕES}

O crescimento micelial dos isolados de $P$. lilacinus teve grande dependência da temperatura de incubação a que foram submetidos, sendo mais rápido a $22,5^{\circ} \mathrm{C}$. Os isolados de $P$. lilacinus revelaram habilidade para infectar os ovos de $M$. paranaensis em meio BDA, principalmente na temperatura de $25^{\circ} \mathrm{C}$.

\section{AGRADECIMENTOS}

Os autores agradecem ao Dr. Rui G. Carneiro, responsável pelo Laboratório de Nematologia do IAPAR, Londrina-PR, pelo fornecimento de inóculo de $M$. paranaensis.

\section{REFERÊNCIAS BIBLIOGRÁFICAS}

ALVES, S. B.; ALMEIDA, J. E. M. de; MOINO JÚNIOR, A.; ALVES, L. F. A. Técnicas de laboratório. In: ALVES, S. B. Controle microbiano de insetos. Piracicaba: FEALQ, 1998. p. 637-711, 1163 p.

CAMPOS, H. D.; CAMPOS, V. P. Efeito da época e forma de aplicação dos fungos Arthrobotrys conoindes, Arthrobotrys musiformis, Paecilomyces lilacinus e Verticillium chlamydosporium no controle de Meloidogyne incognita raça 2 do feijoeiro. Summa Phytopathologica, Piracicaba, v. 22, n. 2, p. 168-171, 1996.

CAMPOS, H. D.; CAMPOS, V. P. Efeito da época e forma de aplicação dos fungos Arthrobotrys conoindes, Arthrobotrys musiformis, Paecilomyces lilacinus e Verticillium chlamydosporium no controle de Meloidogyne exigua do cafeeiro. Fitopatologia Brasileira, Brasília, v. 22, n. 3, p. 361-365, 1997.

CARNEIRO, R. M. D. G. Estude des possibilities d'utilisation du champignon nematophage Paecilomyces lilacinus (Thom.) Samson, 1974, Comme Agent de Lutte
Biologique contre Meloidogyne arenaria (Neal, 1889), Chitwood, 1949. 1986. 119 f. Tese (Doutorado) - Universite des Sciences et Techniques du Languedoc, Paris, 1986.

CARNEIRO, R. M. D. G. Princípios e tendências do controle biológico de nematóides com fungos nematófagos. Pesquisa Agropecuária Brasileira, Brasília, v. 27, p. 113-121, 1992.

CARNEIRO, R. M. D. G.; CARNEIRO, R. G.; ABRANTES, I. M. O.; SANTOS, M. S. N. A.; ALMEIDA, M. R. A. Meloidogyne paranaensis n. sp. (Nemata: Meloidogynidae), a root-knot nematode parasitizing coffee in Brazil. Journal of Nematology, College Park, v. 28, n. 2, p. 177-189, 1996.

COSTA, S. B.; CAMPOS, V. P. Production of Heterodera glycines females in hydroponic solution and pathogenicity tests with fungi isolated from cysts to $H$. glycines and Meloidogyne spp. females. Summa Phytopathologica, Piracicaba, v. 23, n. 3/4, p. 239-243, 1997.

FARIA, M. R.; TIGANO, M. S. Coleção de fungos entomopatogênicos do Cenargen. Brasília, DF: Embrapa, 1996. $76 \mathrm{p}$.

FELLI, L. S.; SACCHI, E. C.; MONTEIRO, A. R. Efeito de Paecilomyces lilacinus, carbamatos e matéria orgânica no controle de Meloidogyne incognita em tomateiro. Nematologia Brasileira, Piracicaba, v. 9, p. 34-35, 1985.

FERRAZ, S.; SANTOS, M. A. Controle biológico de fitonematóides pelo uso de fungos. In: Revisão anual de patologia de plantas. Passo Fundo: Embrapa, 1995. p. 283-314.

FIORETTO, A. M. C.; VILLACORTA, A. Exigências térmicas para o desenvolvimento do fungo nematófago Paecilomyces lilacinus. Pesquisa Agropecuária Brasileira, Brasília, v. 24, n. 8, p. 975-978, 1981.

FREIRE, F. C. O.; BRIDGE, J. Parasitism of eggs, females and juveniles of Meloidogyne incognita by Paecilomyces lilacinus and Verticillium chlamydosporium. Fitopatologia Brasileira, Brasília, v. 10, n. 3, p. 577-596, 1985.

FREITAS, L. G.; FERRAZ, S.; ALMEIDA, A. M. S. Controle de Meloidogyne javanica em tomateiro pela produção de mudas e substrato infestado com Paecilomyces lilacinus. Nematologia Brasileira, Campinas, v. 23, n. 1, p. 65-71, 1999. 
FREITAS, L. G.; FERRAZ, S.; MUCHOVEJ, J. J. Effectiveness of different isolates of Paecilomyces lilacinus and an isolate of Cylindrocarpon destructans on the control of Meloidogyne javanica. Nematropica, Lousiana, v. 25, n. 2, p. 109-115, 1995.

HEWLETT, T. E.; DICKSON, D. W.; MITCHELL, D. J.; KANNWISCHER-MITCHELL, M. E. Evaluation of Paecilomyces lilacinus as a biocontrol agent of Meloidogyne javanica on tobacco. Journal of Nematology, Lakeland, v. 20, n. 4, p. 578-584, 1988.

JATALA, P. Biological control of plant-parasitic nematodes. Annual Review of Phytopathology, New York, v. 24, p. 453489, 1986.

KERRY, B. R. An assessment of progress toward microbial controle of plant parasitic nematode. Journal of Nematology, Lakeland, v. 22, n. 45, p. 621-631, 1990. Supplement.

KERRY, B. R.; CRUMP, D. H.; MULLEN, L. A. Studies of the cereal-cyst nematode, Heterodera avenae Woll. under continuous cereals, 1975-1978: II. fungal parasitism of nematode eggs and females. Annals Applied Biology, [S.1.], v. 100, n. 3, p. 489-499, 1982.

KRZYZANOWSKI, A. A.; FIGUEIREDO, R.; SANTIAGO, D. C.; FAVORETO, L. Levantamento de espécies e raças de Meloidogyne em cafeeiros no estado do Paraná. In: SIMPÓSIO DE PESQUISA DOS CAFÉS DO BRASIL, 2., 2001, Vitória. Anais... Vitória: UFES, 2001. p. 81.

LORDELLO, A. I. L.; LORDELLO, R. R. A.; FAZUOLI, L. C. Levantamento de espécies de Meloidogyne em cafeeiros no estado de São Paulo. In: SIMPÓSIO DE PESQUISA DOS CAFÉS DO BRASIL, 2., 2001, Vitória. Anais... Vitória: UFES, 2001. p. 81-82.
MANKAU, R. Biological control of nematode pests by natural enemies. Annual Review Phytopathology, New York, v. 18 , p. $425-440,1980$.

MIZOBUTSI, E. H.; FERRAZ, S.; RIBEIRO, R. C. F. Avaliação do parasitismo de diversos isolados fúngicos em Heterodera glycines e Meloidogyne javanica. Nematologia Brasileira, Piracicaba, v. 24, n. 2, p. 167-172, 2000.

MOURA, R. M. O gênero Meloidogyne e a Meloidoginose: parte I. In: LUZ, W. C. da; FERNANDES, J. M. C.; PRESTES, A. M.; PICININI, E. C. (Eds.). Revisão anual de patologia de plantas. Passo Fundo: Embrapa, 1996. v. 4, p. 281-315.

NOVARETTI, W. R. T.; DINARDO-MIRANDA, L. L.; TOTINO, L. C.; STRABELLI, J. Efeito da aplicação conjunta do fungo Paecilomyces lilacinus e do nematicida Furadan 5 G no controle de nematóides em cana-de-açúcar. Nematologia Brasileira, Piracicaba, v. 10, p. 133-144, 1986.

RODRÍGUEZ-KÁBANA, R.; MORGAN-JONES, G.; GINTIS, B. O. Effect of chitin amendments to soil on Heterodera glycines, microbial populations and colonization of cysts by fungi. Nematropica, Lousiana, v. 14, p. 10-25, 1984.

SANTOS, M. A. dos; FERRAZ, S.; MUCHOVEJ, J. J. Evaluation of 20 species of fungi from Brazil for biocontrol of Meloidogyne incognita race 3. Nematropica, Lousiana, v. 22, n. 2, p. 183-192, 1992.

SOSA-GOMEZ, D. R. Fungos entomopatogênicos: catálogo de isolados. Londrina: Embrapa-Soja, 2002. v. 1, p. 1-32. (Série Documentos).

STIRLING, G. R.; WEST, L. M. Fungal parasites of rootknot nematode eggs from tropical and sub-tropical regions of Australia. Australasian Plant Pathology, Sidney, v. 20, n. 4, p. 149-154, 1991. 